\title{
COVID-19 Induced Immunosuppression resulting in Pulmonary Mucormycosis : A Long COVID sequalae
}

\author{
Dhruv Talwar ${ }^{1}$,Sunil Kumar ${ }^{2}$,Sourya Acharya ${ }^{3}$,Sparsh Madaan ${ }^{4}$,Vidyashree Hulkoti ${ }^{1}$
}

\begin{abstract}
With increasing incidence of Severe Acute Respiratory Distress Virus 2(SARS -CoV -2) in India, there has been growing reports of super infections in post covid 19 period. Long COVID refers to persistence of COVIDI 9 symptoms for weeks to months. While most common manifestation of long COVID is fatigue and ill health, it may lead to development of life-threatening complications like Pulmonary Mucormycosis. We report a case of 54 year young, non-diabetic previously healthy male who had moderate COVIDI9 treated with low dose steroids and recovered completely, only to present with hemoptysis 3 months later on High Resolution Computed Tomography Scan of the Chest and Rhizopus fungi on bronchoalveolar lavage confirming Post Covid Pulmonary Mucormycosis in non-immunocompromised host. Patient was managed with dual antifungal therapy intravenous Liposomal Amphotericin B and Posaconazole for 3 weeks, followed by oral Posaconazole.
\end{abstract}

Keywords: Pulmonary Mucormycosis, Long Covid, Immunosuppression

(c) (i) (2)

DOI: https://doi.org//0.3329/jom.v22i2.56707 Copyright: () 202I Talwar D. This is an open access article published under the Creative Commons Attribution-NonCommercial-NoDerivatives 4.0 International License, which permits use, distribution and reproduction in any medium, provided the original work is properly cited, is not changed in any way and it is not used for commercial purposes.

Received: I/ June, 202I;

Accepted: 23 June 202I

1 Post Graduate Resident, Department of Medicine, Jawaharlal Nehru Medical College, Datta Meghe Institute of Medical Science, Sawangi meghe, Wardha, Maharashtra, India

2 Professor, Department of Medicine, Jawaharlal Nehru Medical College, Datta Meghe Institute of Medical Science, Sawangi meghe, Wardha, Maharashtra, India

3 Professor and Head of Department, Department of Medicine,Jawaharlal Nehru Medical College, Datta Meghe Institute of Medical Science, Sawangi meghe, Wardha, Maharashtra, India

4 Post Graduate Resident, Department of Obstetrics and Gynaecology, Jawaharlal Nehru Medical College, Datta Meghe Institute of Medical Science, Sawangi meghe, Wardha, Maharashtra,India 442001

Corresponding Author: Dr Sunil Kumar, Professor, Department of Medicine, Jawaharlal Nehru Medical College, Datta Meghe Institute of Medical Science (Deemed to be University), Sawangi Meghe, Wardha, Maharashtra, India 442001, Email id-sunilkumar med@gmail.com

\section{Introduction}

Severe Acute Respiratory Distress Virus 2(SARS-CoV-2) a novel coronavirus has perplexed the health care professionals around the world since last year with its ever changing presentations and no specific treatment ${ }^{[1]}$. Adding to the burden of ongoing pandemic, there has been an emergence of Long Covid Syndrome which refers to persistence of symptoms following acute infection with COVID-19. Although, there is difference of opinion regarding definition of long COVID however National Institute for Health and Care Excellence (NICE) have defined long COVID as clinical manifestations 12 weeks after getting infected with COVID-19. Another term used to describe manifestations of symptoms presenting after 12 weeks of after COVID-19 is Post COVID Syndrome whereas 'Ongoing COVID-19 infection' is defined as symptoms which present during four weeks to twelve weeks following infection with COVID-19. Most common presenting features of Long Covid syndrome are fatigue, muscle pain, loss or distortion of smell along with low grade fever. However since the first 
wave of novel Coronavirus, rise of secondary infections has been reported worldwide. With ever increasing cases of COVID-19, there has been upcoming research for treatment options in the absence of a definitive treatment. ${ }^{2}$ As per evidence based treatment modality which seems to work in moderate to critical COVID-19 is systemic corticosteroids but carries a major side effect of immunosuppression. Last wave left us with huge burden of diabetes post use of corticosteroids and associated super infections. Use of immunomodulators, using monoclonal antibodies to target immune dysgenesis in covid has further complicated the scene with rare infections. Combined use of corticosteroids and immunomodulators along with covid immunosuppression has led to triple whammy for infectious complications with rise of opportunistic fungal infections like mucormycosis and candidiasis. Mucormycosis has imposed significant impediment in battle against the ongoing pandemic during second wave in India. Most common presentation of Mucor is Rhino orbital mucormycosis. ${ }^{3}$ Pulmonary mucormycosis is a rare complication of Long Covid but unrecognized and untreated it carries very high mortality. We report a case of 54 year old male with no prior co morbidities who presented three months following moderate COVID19 infection with the complaints of haemoptysis and was promptly diagnosed as a case of pulmonary mucormycosis. This reflects importance of high index of suspicion to diagnose an infrequent complication of emerging Long Covid Syndrome even when there is no history of diabetes or use of immunosuppressive drugs during acute COVID.

\section{Case Report}

A 54 year old male presented with the chief complaint of haemoptysis since two days with dry cough for one week. There was history of low grade intermittent fever during this one week He had history of COVID-19 three months back which was confirmed by reverse transcriptase polymerase chain reaction when his HRCT Chest showed bilateral ground glass opacities and consolidations with a CT Severity Score of 10/25 and was given remdesavir, systemic steroids ( MPS $40 \mathrm{mg}$ /day for 5 days) along with other supportive treatment. He received tapering oral steroids for another 5 days post discharge and remained mostly asymptomatic since then. He had no history of diabetes mellitus, hypertension or any malignancy in the past and his blood sugars during hospitalization were normal. He gave no history of immunosuppressive treatment in the past. On examination patient was conscious and oriented, his pulse was 104 beats per minute regular in rhythm and volume, blood pressure was $130 / 86 \mathrm{~mm}$ hg in right arm supine position and spo 2 was 93 percent on room air with respiratory rate of 26 breaths per minute.
Table 1. Showing lab investigations of the case

\begin{tabular}{|c|c|}
\hline Lab Parameter & Result \\
\hline Interleukin 6 & $98 \mathrm{pg} / \mathrm{ml}$ \\
\hline Serum Ferritin & $588 \mathrm{~g} / \mathrm{ml}$ \\
\hline Lactate Dehydrogenase & $600 \mathrm{u} / \mathrm{ml}$ \\
\hline CRP & $67 \mathrm{mg} / \mathrm{l}$ \\
\hline Complete Blood Count & $\begin{array}{l}\text { Haemoglobin } 13.7 \mathrm{gm} / \mathrm{dl} \\
\text { MCV } 88 \mathrm{fl} \\
\text { Platlet count } 92000 / \mathrm{dl} \\
\text { White Blood Cell Count 9200/dl }\end{array}$ \\
\hline Kidney Function Test & $\begin{array}{l}\text { Creatinine: } 1.3 \mathrm{mg} / \mathrm{dl} \text {, } \\
\text { Urea } 29 \mathrm{mg} / \mathrm{dl}, \\
\text { Sodium } 134 \mathrm{mmol} / 1 \text {, } \\
\text { Potassium }-3.7 \mathrm{mmol} / 1\end{array}$ \\
\hline Liver function Test & $\begin{array}{l}\text { Total Protein- } 6.2 \mathrm{gm} / \mathrm{dl} \text {, } \\
\text { Albumin2.6gm/dl, } \\
\text { Globulin2.6gm/dl, } \\
\text { aspartate aminotransferase } \\
27 \text { units/1, alanine aminotrans- } \\
\text { ferase } 38 \text { units/1, } \\
\text { Alkanline Phophatase } 103 \mathrm{IU} / 1 \text {, } \\
\text { Total Bilirubin }: 1.1 \mathrm{mg}\end{array}$ \\
\hline $\mathrm{HbA} 1 \mathrm{c}$ & 5.5 \\
\hline
\end{tabular}

Examination of head and neck, oral cavity was unremarkable. On examination of chest there were crackles noticed over right mammary region, heart sounds were normal and abdomen was soft non tender with no hepatosplenomegaly. HRCT Chest was done which showed consolidation of right upper lobe with atypical cavitation's and 'Reverse Halo Sign' (figure 2) suggestive of infective aetiology (? Fungal). Bronchoscopy was performed and Bronchial Alveolar Lavage was sent for smear and bacterial, tubercular and fungal cultures. BAL smear showed broad, aseptate hyphae and was confirmed as mucor species clinching the diagnosis of Pulmonary Mucormycosis (Figure 3). CT scan of paranasal sinus was performed which showed no obvious abnormality suggestive of mucormycosis (Figure 4). Lab investigations are mentioned in table 1 and showed normal HbAlc levels ruling out diabetes mellitus as a risk factor. Patient was started on dual antifungal treatment with parental Liposomal Amphotericin B along with Posaconazole and discharged. $\mathrm{He}$ is currently undergoing treatment with oral Posaconazole and is being monitored for progress. 


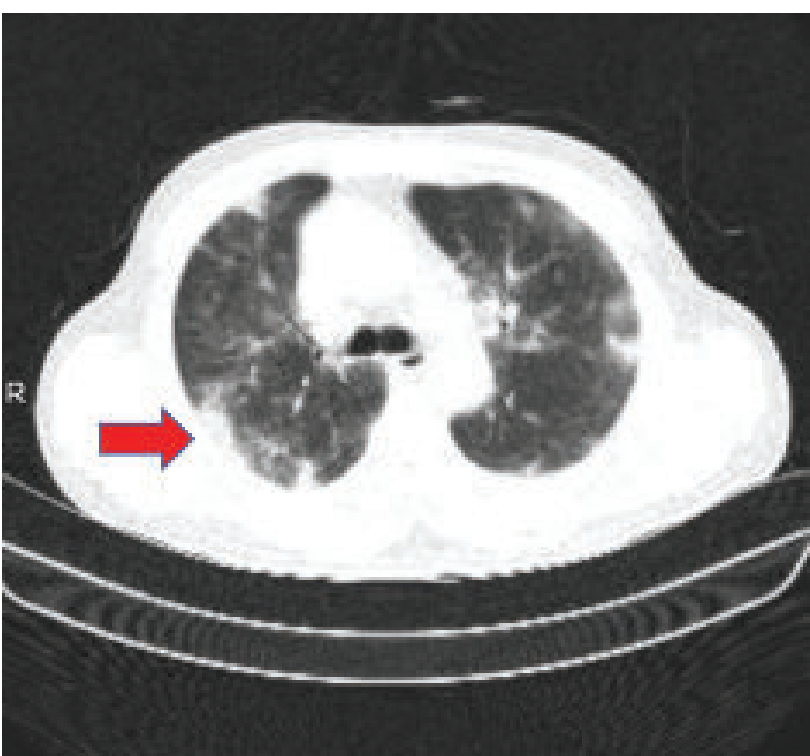

Figure 1: Showing patients HRCT Chest from three months back with bilateral ground glass opacities

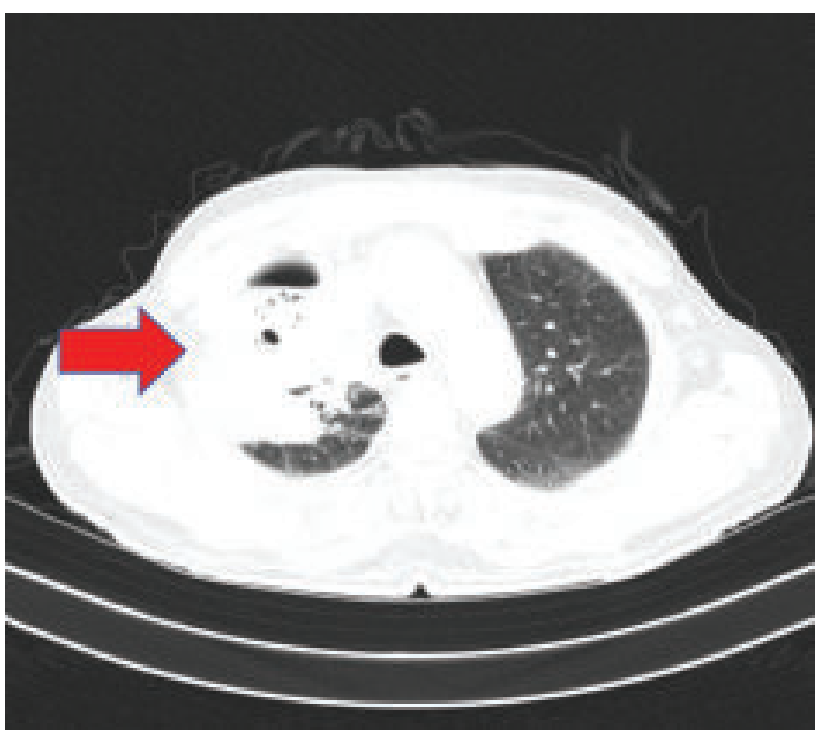

Figure 2: Present HRCT Chest showing Reverse Halo Sign suggestive of pulmonary mucormycosis

\section{Discussion}

Mucormycosis is an opportunistic fungal infection caused by fungi belonging to moulds known as mucorales. Generally species involved are Mucor, Rhizopus, Absidia and Cunninghamella. Hyphae growing around blood vessels are a characteristic feature of mucormycosis and it is invariably fatal disease in the immunocompromised and diabetic individuals unless detected early and treated aggressively. Mucormycosis is now known to have six different presentations including rhino orbital, cutaneous, pulmonary, gastrointestinal, central nervous system and miscellaneous

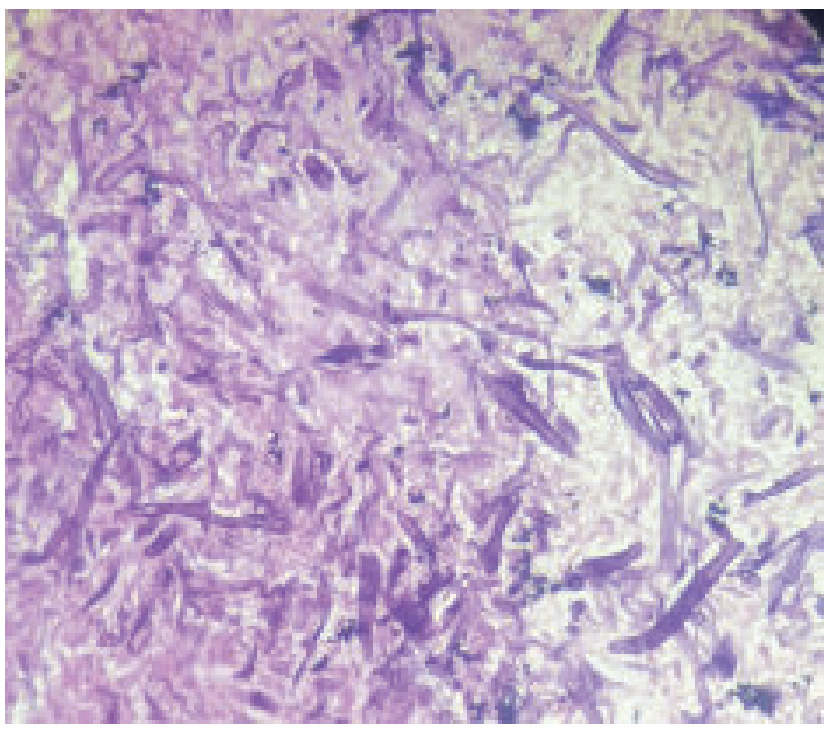

Figure 3: Showing Growth of Mucor Species on culture of Bronchial Alveolar Lavage

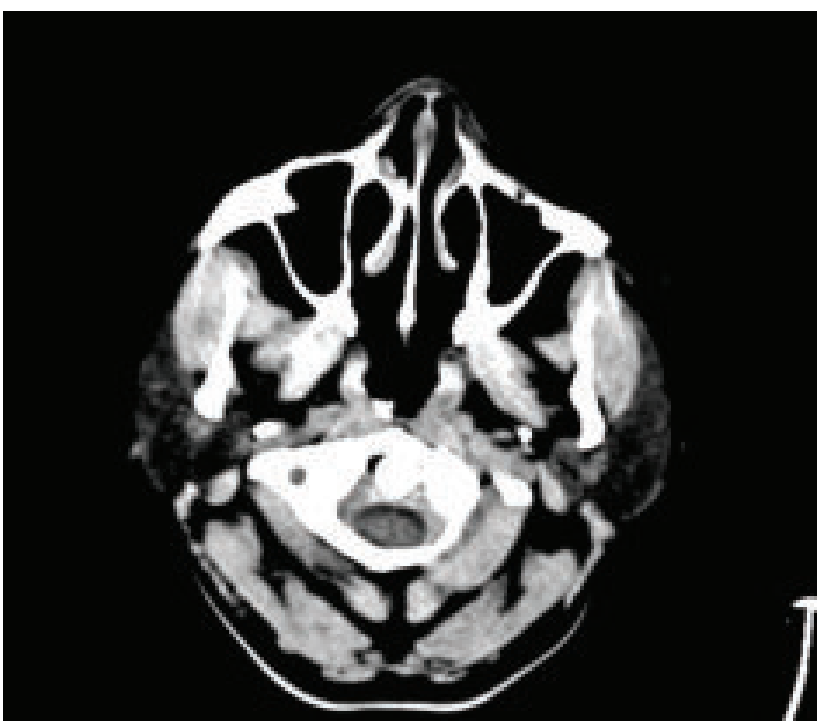

Figure 4: Showing CT of Para Nasal Sinus showed mild mucosal thickening in left maxillary sinus with no obvious abnormality suggestive of mucormycosis.

including the bone. breast and kidneys. While rhino orbital mucormycosis is commonly associated with COVID19 but pulmonary mucormycosis is expected in view of uncontrolled sugars, steroids and immunomodulators use but presentation in non-diabetic who did not receive immunomodulators, currently not on steroids is very unlikely. The first case of pulmonary mucormycosis was reported by Fubringer in the year 1876. Risk factors for pulmonary mucormycosis includes diabetes mellitus, immunocompromised patients including patients on chemotherapy and patients with hematological malignancies like leukemia or lymphoma, metabolic acidosis, 


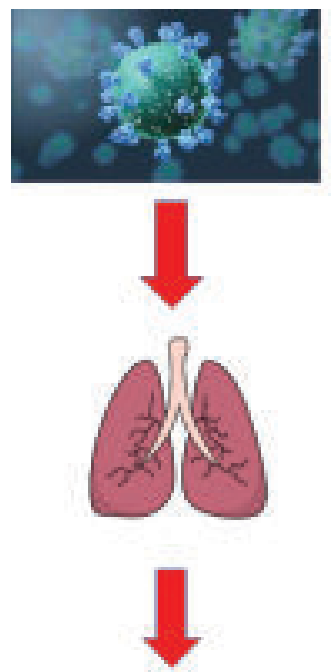

Disruption of tight junction

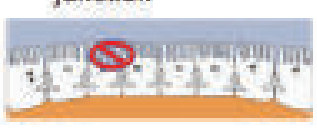

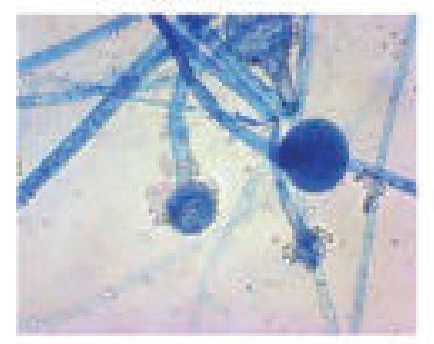

PULMONARY MUCORMYCOSIS

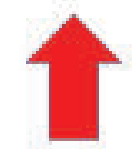

Compromised Immunity
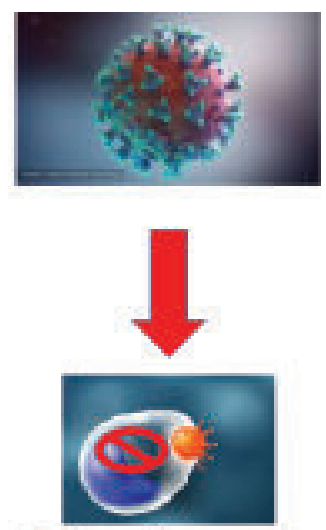

Defective Phagocytosis and Downregulation of Complement System

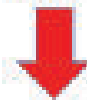

Inadequate Deferse against COVID19 and other secondary infections

Figure 5: Pathophysiology of pulmonary mucormycosis in COVID19

agammaglobulinemia and post solid organ transplantation. Mucormycosis is spread via inhalation of fungal spores or through inoculation over the skin. In conditions such as diabetes there is hyper glycation of iron sequestering protein leading to a release free iron and upregulation of CGRP 78 receptor which ultimately leads to increased expression of $\mathrm{COTH}$ protein and increased fungal binding. The inhaled spores are phagocytosed by immune cells thus protecting against fungal infections however in conditions like neutropenia and high dose corticosteroids there is decreased phagocytosis leading to fungal proliferation and invasion of blood vessels. Hemoptysis typically occurs with vascular invasion and is a serious symptom and it may prove to be fatal. Radiologically, diagnosis of pulmonary mucormycosis is suggested by the reverse halo sign which signifies ground glass opacity which is surrounded by consolidation and atypical cavitation. A similar sign known as halo sign is seen in pulmonary aspergillosis where there is nodule/ consolidation surrounded by a ground glass opacity in its periphery. ${ }^{4}$ Diagnosis of pulmonary mucormycosis can be established by a CT guided biopsy or a bronchoalveolar lavage taken by the means of a bronchoscopy. Smear examination shows typical broad aseptate hyphae which on culture shows growth of fungal moulds causing mucormycosis (Mucor, Rhizopus, absidia and cunninghamella). Once the diagnosis is established treatment is initiated by the means of dual antifungal therapy. Amphotericin B remains the gold standard drug against mucormycosis and it can be supported by posaconazole. ${ }^{5}$ Surgical treatment in the form of lobectomy,wedge resection and pneumonectomy is often required to support medical treatment. ${ }^{6}$

Another aspect of debate in our case of COVID associated mucormycosis is if dysregulation of the immune system by COVID19 can solely lead to development of this opportunistic infection and if so, how long this risk lasts. Ingress of infecting agents is explained by the natural barrier of tight junctions in the alveolar epithelium being disrupted by the SARS CoV2, due to down regulation of proteins involved in tight junction formation by the SARS CoV2 including proteins ZO-1 and 2, Claudin 2,3,11 and 19, cingulin,Afadin,Rho GTPase-activating protein 17 and cAMP-dependent protein kinase catalytic subunit alpha (PRKACA.Thus, SARS-CoV-2 alters the formation and functioning of the tight junction damaging the physical barrier against invasion by environmental pathogens predisposing patients to develop secondary or super infections (Figure 5). Additionally, there is downregulation of the complement system with dysregulation of phagocytosis due to decrease in tyrosine kinase levels in the spleen which is involved in phagocytosis in COVID-19 causing significant immunosuppression further prejudicing 
them to developed secondary opportunistic infections ${ }^{[7] .}$ Role of increase ferritin in covid 19 and use of nasal cannula to provide high flow oxygen and tissue hypoxia are all being considered as attributable factors to development of mucormycosis. But., these need further evaluation and studies.

In our case the patient was a healthy male with no prior co morbidities and had a normal HbA1c, no prolonged or high dose systemic steroids use with no prior use of immunomodulators as risk factors most commonly being associated with pulmonary mucormycosis. Additionally, the patient reported 3 months after being tested RT PCR positive to COVID 19, highlighting need for constant vigil despite good and adequate recovery in this new age disease. Although, in our case scenario it is reasonable to conclude that COVID19 was the sole major risk factor for immune dysregulation predisposing the patient to develop a rare opportunistic infection in the form of pulmonary mucormycosis, emphasizing need for careful follow up of patients in long Covid.

\section{Conclusion}

We conclude that although Pulmonary Mucormycosis is very rare in the absence of risk factors but SARS CoV2 may alter the immune system of an individual thereby predisposing them to secondary opportunistic infections even months after recovery. Also, rhino orbital mucormycosis is most common presentation of mucormycosis frequently reported during this second wave of COVID 19and has become a notifiable disease in India but pulmonary mucormycosis may also be seen as a rare complication of long covid hence the treating physicians should keep high index of suspicion for opportunistic infections as immunosuppression may take much longer to recover. Pulmonary mucormycosis during the long covid syndrome would test skills of physicians and this case illustrates importance of early diagnosis and prompt treatment.

\section{References:}

1. Jain A ,Talwar D ,Kumar S. Spectrum of Respiratory Involvement in COVID 19 Era; An Overview. Indian J of Forensic Med \& Toxicol. 2020;14(4):6593-9.

2. Talwar D, Kumar S, Madaan S, Khanna S, Annadatha A. Intractable Singultus: Atypical presentation of COVID 19. Medical Science, 2021, 25(111), 1183-1187

3. Dronamraju S, Nimkar S, Damke S, Agrawal S, Kumar S. Angioinvasion of anterior cerebral artery by rhinocerebral mucormycosis leading to intraparenchymal hemorrhage: A rare case report. Medical Science, 2021, 25(111), 1088-1091

4. Garg D, Muthu V, Sehgal IS, Ramachandran R, Kaur H, Bhalla A, Puri GD, Chakrabarti A, Agarwal R. Coronavirus Disease (Covid-19) Associated Mucormycosis (CAM): Case Report and Systematic Review of Literature. Mycopathologia. 2021 May;186(2):289-298.

5. Arastehfar A, Carvalho A, van de Veerdonk FL, Jenks JD, Koehler P, Krause R, et al. COVID-19 associated pulmonary aspergillosis (CAPA)-from immunology to treatment. J Fungi (Basel). 2020;6(2):91.

6. Kumar G, Adams A, Hererra M, Rojas ER, Singh V, Sakhuja A, et al. Predictors and outcomes of hais in COVID-19 patients. Int J Infect Dis. 2020;104(3):287-92

7. Kimmig LM, Wu D, Gold M, Pettit NN, Pitrak D, Mueller $\mathrm{J}$, et al. IL-6 inhibition in critically Ill COVID-19 patients is associated with increased secondary infections. Front Med (Lausanne) 2020;7:583897. 